\title{
Aspects of energetic substrate metabolism of in vitro and in vivo bovine embryos
}

\author{
D.K. de Souza ${ }^{1,2}$, L.P. Salles ${ }^{1,3}$ and A.A.M. Rosa e Silva ${ }^{1}$ \\ ${ }^{1}$ Laboratório de Biotecnologia da Saúde, Faculdade de Medicina, Universidade de Brasília, Brasília, DF, Brasil \\ ${ }^{2}$ Faculdade da Ceilândia, Universidade de Brasília, Brasília, DF, Brasil \\ ${ }^{3}$ Departamento de Biologia Molecular, Instituto de Biologia, Universidade de Brasília, Brasília, DF, Brasil
}

\begin{abstract}
Although the metabolism of early bovine embryos has not been fully elucidated, several publications have addressed this important issue to improve culture conditions for cattle reproductive biotechnologies, with the ultimate goal of producing in vitro embryos similar in quality to those developing in vivo. Here, we review general aspects of bovine embryo metabolism in vitro and in vivo, and discuss the use of metabolic analysis of embryos produced in vitro to assess viability and predict a viable pregnancy after transference to the female tract.
\end{abstract}

Key words: Bovine pregnancy rates; Embryo metabolism; Metabolism profile; In vitro fertilization; Embryo culture conditions; In vivo embryo

\section{Introduction}

In vitro production of bovine embryos is a process that depends on the provision of nutrients for the gametes and preimplantation embryos $(1,2)$. The quality of oocytes and spermatozoids is a determinant of embryo competence, but the nutrition of the embryo is one of the most important influences of embryo viability.

Despite the presence of glycogen and triglycerides as energy reserves in the bovine embryo, the viability of embryonic cells in culture is maintained primarily by energetic substrates present in the culture medium. The balance of nutrients supplied to the embryo in vitro and the intrauterine environment can both affect the phenotypic characteristics observed in adult life (3).

An example of the consequences of cell modifications that occur during in vitro culture is the 'large offspring syndrome' characterized by lower pregnancy and higher abortion rates, oversized offspring, abnormal placentas, and other pregnancy dysfunctions in cattle embryos produced by in vitro culture (4). If culture environments can influence the development of embryos and the health of calves, the analysis of culture nutrient supplements is an essential goal to improve bovine production and prevent inappropriate phenotypes. However, the conditions corresponding to under- or over-nutrition in the environment of embryo cultures and how they can influence phenotypic characteristics have not yet been precisely determined.
The analysis of metabolite consumption and production, which can be determined from samples of culture medium, is correlated with the viability of embryos in culture (5). The noninvasive technique of analyzing culture medium metabolites can be used by researchers to identify in vitro embryo profiles related to healthy future offspring. Analysis of the metabolism of embryos produced in vitro also contributes to understanding of a culture environment adequate to allow embryos to reach competence (6).

In the last few years, numerous publications have addressed the metabolic profiles of embryos produced in vitro (5-13), but no consensus has been reached on the metabolic status and concentration of energetic substrates capable of improving the efficiency of in vitro fertilization (IVF). It is well known that in vivo embryos have a 'quiet' metabolism that seems to affect their viability $(5,6)$ - that is, a low metabolic and glycolytic rate and amino acid turnover in addition to high antioxidant capacity $(5,6)$. A culture system that allows in vitro embryos to have a metabolism similar to those in vivo (i.e., naturally quiet) is required. However, the provision of adequate substrates in the preimplantation stages has not yet been achieved.

Numerous publications have evaluated metabolic markers of embryo viability utilizing invasive techniques such as real-time PCR that prevent embryo transfer. In this paper, we discuss the metabolic profile of bovine

Correspondence: D.K. de Souza: <dany.kaiser@gmail.com>; A.A.M. Rosa e Silva: <aamresil@unb.br>.

Received February 1, 2014. Accepted June 16, 2014. First published online January 23, 2015. 
embryos and the data produced using noninvasive methods to measure energy metabolism. We also highlight possible correlations between the quiet metabolic profile of bovine embryos in vivo and profiles produced in vitro in order to improve embryo development.

\section{General aspects of bovine embryo metabolism}

During the initial stages, the transcriptional ability of embryos is limited until embryonic genome activation, which occurs at the 8-cell stage in bovine embryos $(7,8)$, demonstrating the relevance of oocyte quality and the embryo's ability to metabolize its own reserves and external energetic substrates (7-9).

Oocyte quality influences cattle blastocyst development, as described by Gulart and Rosa e Silva (unpublished data from our laboratory). The in vitro maturation of the cumulus-oocyte complex in a medium supplemented with several hormones reduced blastocyst production in vitro. On the other hand, a simple medium without hormones allowed the production of blastocysts at the same rate found after culture in a commercial medium (around 20-30\%). These important data corroborate the quiet hypothesis, demonstrating that less stimulation relates directly to better development, but does not negate the importance of the embryo-culture environment in rescuing embryos from cell apoptosis.

The consumption of glucose, pyruvate, lipids and amino acids is responsible for ATP synthesis in the embryonic stem cells. Bovine embryos also have glycogen and lipid reserves; however, the glycogen concentration seems almost negligible and is poorly described in the literature (10). Lipids represent the most abundant energy reservoir of bovine embryos (11).

In mammalian cells, ATP generation occurs via oxidative pathways or by glycolysis. Glycolysis occurs in the cytoplasm, does not require oxygen for enzymatic metabolic reactions, and generates lactate and only four ATP molecules per molecule of glucose oxidized. The oxidative pathway occurs in mitochondria, necessarily requires oxygen, and is able to oxidize pyruvate completely, generating $\mathrm{CO}_{2}, \mathrm{H}_{2} \mathrm{O}$, and $30-32$ molecules of ATP via the Krebs' cycle and oxidative phosphorylation enzymes (Figure 1). These pathways are interdependent and necessary for viable embryonic development (12), and the oxidative pathway is the most important one for ATP production (13). For example, glycolysis is responsible for only 2.6$8.7 \%$ of the ATP generated by porcine embryo cells, depending on the embryonic preimplantation phase (13).

The energetic metabolism between the zygote and the 8-cell stage depends on pyruvate, glucose and oxygen, with relatively little consumption of these $(14,15)$ (Figure 2). The precompaction stage is characterized by $93-96 \%$ ATP produced from the oxidative pathway, but this decreases to $82 \%$ in the compaction process (14). In addition, pyruvate is essential for the first embryonic cleavage; it is also an important energetic substrate for ATP generation in cells with a low glycolytic rate and for intracellular $\mathrm{pH}$ regulation (14).

Pyruvate and glucose are consumed in low amounts until the 16-cell stage $(14,15)$. Glucose, pyruvate and oxygen consumption increases significantly with morula compaction and blastocyst formation (Figure 2), indicating an increase in energy requirements, with oxidative metabolism detected by $\mathrm{CO}_{2}$ production $(14,15)$. Blastocyst formation and the cavitation process significantly increase energy demands, the consumption of glucose, pyruvate, and oxygen (Figure 2), and the synthesis of protein $(14,15)$. The combination of glucose, lactate and pyruvate in the culture medium significantly reduces glucose metabolism in vitro (15).

Lactate production also increases during embryo development, primarily in the blastocyst stage $(14,15)$, and all these data indicate high rates of aerobic glycolysis. When lactate is used as the only energetic substrate, compared to pyruvate or glucose alone, it is clear that glucose and pyruvate are preferred and generate more $\mathrm{CO}_{2}$ than lactate (15). However, the most consumed energetic substrate in vitro, i.e., glucose or pyruvate, remains obscure when both are present in the medium, and obviously depends on the concentration of each one $(14,15)$.

All cells have their own intracellular pool of amino acids and relevant roles in embryo development $(16,17)$. Amino acids are highly consumed between the 8-cell and compact morula stages owing to protein, ATP and signaling molecule synthesis, and osmoregulation and $\mathrm{pH}$ regulation (17). Mouse embryos are differently regulated by the amino acid profile of culture medium (18), demonstrating the relevance of the amino acid composition for blastocyst development.

The interrelation of glucose/pyruvate and amino acids is also evident. Pyruvate is one of the molecules responsible for ammonium detoxification, via the synthesis of alanine, which can be released into the extracellular medium (16).

\section{Blastocyst: the embryo stage with the highest energy consumption rates}

The metabolic demands and nutrient consumption increase between morula compaction and blastocyst formation (14), measured by oxygen, glucose, and pyruvate consumption (Figure 2) and lactate production $(13,14,17)$. The development of the blastocyst cavity is directly mediated by increased activity of the $\mathrm{Na}^{+} / \mathrm{K}^{+}$ATPase pump, which generates sodium and water (osmosis) influx for cavity formation (19). During this process, $86 \%$ of ATP production results from oxidative phosphorylation pathway (20).

Embryonal mitochondria originate from the oocyte and are described as 'immature' at the initial phases of embryo development, displaying a round or ovoid shape, with few 


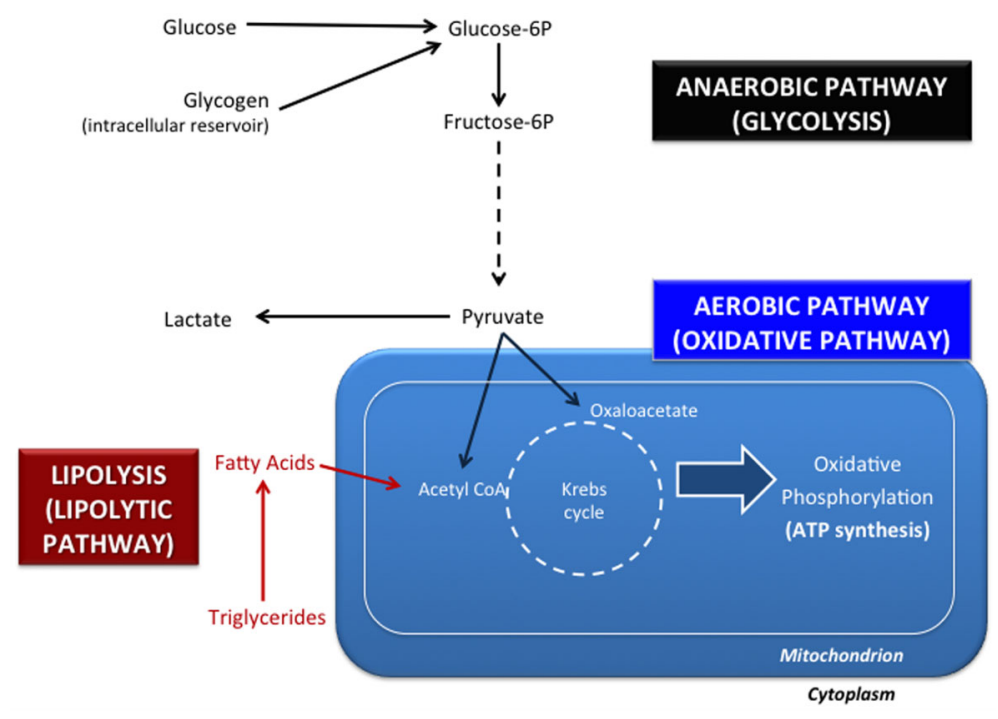

Figure 1. Essential metabolic pathways for bovine embryonic development. The anaerobic pathway (glycolysis or the glycolytic pathway) does not utilize oxygen and generates lactate. Glucose, one of the most important metabolic substrates for glycolysis, is obtained from the extracellular medium or from glycogen reservoirs. In the aerobic (or oxidative) pathway, pyruvate is converted into oxaloacetate or acetyl-CoA and fatty acids are converted into acetyl-CoA. ATP synthesis is dependent on the Krebs' cycle and on oxidative phosphorylation (enzymatic reactions catalyzed by proteins from the inner mitochondrial membrane). The lipolytic pathway (or lipolysis) allows the oxidation of fatty acids, obtained from lipid (triglyceride) reservoirs or from the extracellular medium.

inner cristae (21). Between the 4- and the 16-cell embryo stages, mitochondria elongate, and the number of cristae starts to increase and continues increasing throughout blastocyst development, concomitantly with higher glucose and pyruvate uptake $(12,14)$. Some researchers have estimated that only $10 \%$ of glucose is completely oxidized

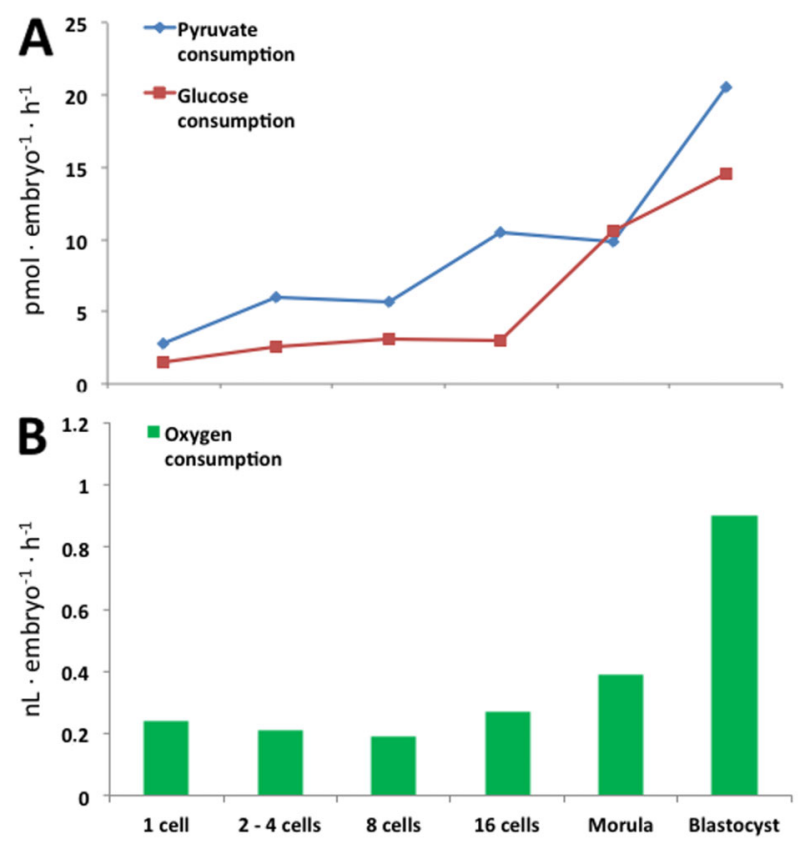

Figure 2. Glucose, pyruvate and oxygen consumption during embryo preimplantation stages. Measurements were performed in isolated in vitro embryos (adapted from Refs. 13 and 14, with permission). until the 16-cell stage, and the glucose oxidation rate increases in subsequent stages $(12,21)$.

During bovine blastocyst development, the activity of $\mathrm{Na}^{+} / \mathrm{K}^{+}$ATPase pump and oxygen consumption are related to the cavitation process, as mentioned before (19). ATP production in bovine embryo is around $459 \mathrm{pmol} \cdot$ blastocyst $^{-1} \cdot \mathrm{h}^{-1}$, and $36 \%$ and $15 \%$ of blastocyst ATP is used for $\mathrm{Na}^{+} / \mathrm{K}^{+}$ATPase pump activity on days 7 and 8 of blastocyst development, respectively (22). The expanding blastocyst reaches $94.5 \mathrm{pmol} \cdot \mathrm{embryo}^{-1} \cdot \mathrm{h}^{-1}$ of pump activity while the other stages maintain around 20-30 $\mathrm{pmol} \cdot$ embryo $^{-1} \cdot \mathrm{h}^{-1}(22)$.

The blastocyst consists of trophoblast/trophectoderm and the inner cell mass. The study of isolated cells demonstrated that the two cell types have different metabolic profiles. Isolated trophoblast cells consume higher levels of pyruvate and produce lactate, while isolated cell from the inner cell mass consume more glucose (23). Mouse blastocyst data also indicate that isolated trophectoderm cells consume more oxygen, produce more ATP, and have a larger number of mitochondria than inner cell mass cells (24), indicating preferential oxidative metabolism. However, it is noteworthy that intact blastocysts have a similar metabolic profile to that of isolated cells from the inner cell mass (23), indicating that this kind of results may be, in part, an artifact of the cells' isolation.

Bovine embryo development is also affected by the presence of amino acids and proteins, such as bovine serum albumin (BSA), in the culture medium. The substitution of BSA by polyvinyl alcohol (PVA) reduces cleavage and blastocyst development rates, as well as blastocyst cell numbers (25). The turnover of amino acids is also altered by the substitution of BSA for PVA, because BSA can be endocytosed by trophoblast cells 
and its degradation generates a specific pool of amino acids, including leucine, lysine and glutamate $(25,26)$, which are related to adequate embryonic development.

Alanine is an important amino acid for embryo development and has the highest levels of release into the culture medium of all the amino acids $(19,23,25)$. Alanine release is related to the cellular excretion of ammonium $(19,23,25)$. The absence of pyruvate in the medium reduces amino acid turnover and alanine production by the embryo (25), indicating the likelihood that ammonium can be transferred to pyruvate and excreted into the extracellular compartment as alanine. Glutamate may also be involved in ammonium detoxification by combining with an ammonium molecule and being converted to glutamine, which can be excreted by the embryo into the extracellular medium (25).

Isolated inner cell mass and trophoblast cells have different amino acid turnover profiles (23). Both blastocyst cell types consume aspartate, arginine, and leucine from the culture medium, and produce and excrete alanine. However, isolated cells from the inner cell mass consume asparagine, glycine, threonine, tyrosine, tryptophan, and phenylalanine, while the same amino acids are produced from isolated trophoblast cells (23). Conversely, trophoblast cells consume methionine, valine, isoleucine, glutamate, serine, histidine, and glutamine, while these amino acids are produced by the inner cell mass (23). All these data are suggestive of metabolic cooperation between the two blastocyst cell types in bovine embryos (23), but again, may be an artifact of the isolation of cell types.

Proteins and amino acids are thus some of the most relevant nutrients for embryo development, and the turnover of amino acids in the culture medium is indicative of embryo viability in vitro $(25,27)$. A profile characterized by a low rate of amino acid turnover during transition of the bovine zygote to the 2-cell embryo has been shown to predict which embryos will develop to the blastocyst stage more often in vitro. That is, $30-35 \%$, on average, of lowmetabolizing embryos reach blastocyst stage in contrast to less than $5 \%$ of high-metabolizing embryos (27).

Lipids and the intracellular nutrient reservoirs are also important for embryonic development. Embryo cells have their own energy storage for ATP generation, including glycogen (8) and triglyceride (9) reservoirs. Although the physiological function of glycogen in embryos has not been well defined, triglycerides represent the major intracellular reservoir of energy in bovine embryos and are synthesized during oocyte maturation (28). Bovine embryos freshly isolated from the female tract have $33.0 \pm 0.7 \mathrm{ng}$ triglycerides per embryo (9).

In the absence of serum, the triglyceride content of embryos is not modified throughout development in vitro, and it is very similar to that observed in vivo (9); however, the importance of lipids for embryo development should not be ignored $(9,13,28)$ because the beta-oxidation of each palmitate fatty acid molecule generates 108 ATP molecules (Figure 3).

Ketone bodies derived from lipids can also be utilized as energetic substrates. Previous studies have shown that in the absence of extracellular nutrients, embryos progressed through only three cleavage divisions (28), but, when cultivated with the fatty acid derivatives acetoacetate and $\beta$-hydroxybutyrate, embryos are able to reach the blastocyst phase $(29,30)$.

Supplementation of embryo culture media with Lcarnitine, an important molecule in the fatty acid oxidation cycle (Figure 3), allowed embryo development to proceed beyond the blastocyst stage, while inhibitors of fatty acid oxidation counteracted this effect (31). Also, in the presence of carbohydrates, the addition of $5 \mathrm{mM} \mathrm{L-}$ carnitine to the medium increased morula and blastocyst formation rates (31), and the inhibition of lipid betaoxidation during oocyte maturation decreased embryo cleavage and blastocyst rates, as well as the number of blastocyst cells per embryo. Inhibition of beta-oxidation decreased oxygen consumption in the 5- and 8-cell stages, and might be directly related to a decrease in blastocyst production rates (28). Overall, these results support the idea that lipid resources comprise the most important energetic reservoir for bovine embryos, that triglycerides and ketone bodies are utilized to generate ATP and sustain embryo development in vitro, and that the inhibition of beta-oxidation reduces embryo development.

Despite the data described here, the pregnancy rate reaches only around $39 \%$, as the indicated ideal concentration of each component, and the combination of multiple medium substrates, do not seem adequate to support embryo development (1). The importance of the combination of energetic supplementation of the medium and intracellular reservoirs is also poorly described in literature. There is no consensus about the ideal composition of the medium. However, we know that embryos having a 'quiet' metabolism are more likely to reach the blastocyst stage (27).

\section{Metabolic profile of in vivo vs in vitro embryos}

Embryos produce more lactate and have higher oxidative rates in vitro than in vivo, which is suggestive of higher metabolic rates in vitro $(5,6,15)$. Also, when in vivo embryos are exposed to extrauterine media, glycolysis and $\mathrm{CO}_{2}$ production increase, demonstrating that culture conditions induce higher metabolic rates (5). This has been described in bovine embryos, where blastocysts in vitro have higher glycolytic rates than in vivo, indicating that culture media might be stressful or might modify embryo metabolism to produce an abnormal profile $(5,14,32)$.

The amino acid turnover (depletion and appearance) of in vivo bovine embryos is also significantly less than that of in vitro embryos (27). We also know that low rates of amino acid turnover during cleavage of zygotes to form 


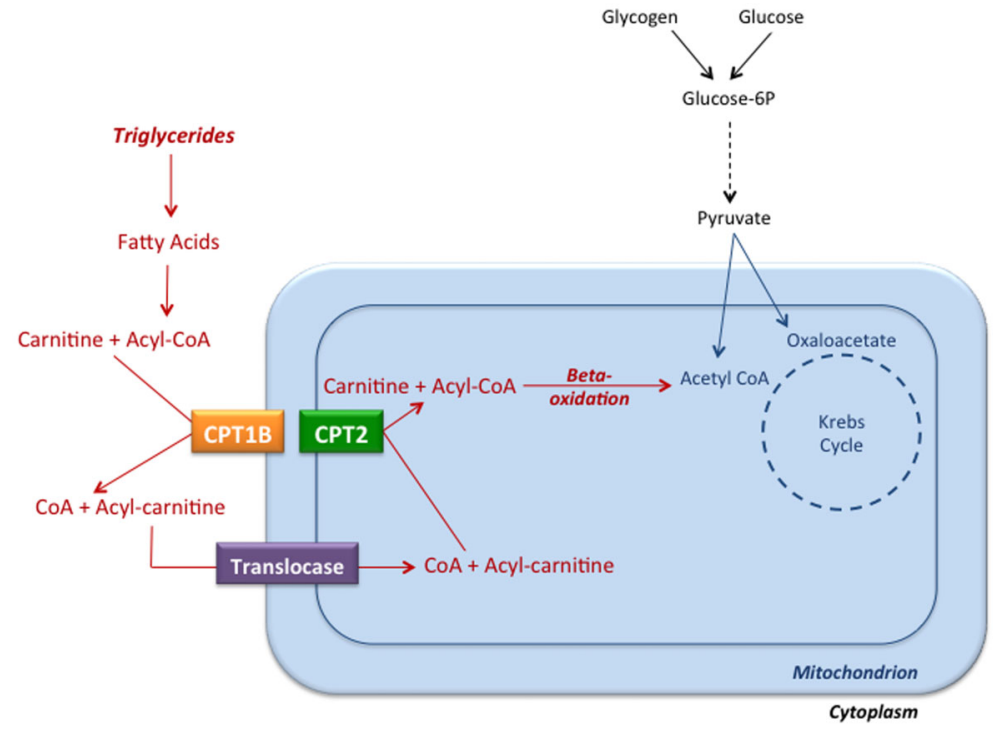

Figure 3. Entry of long-chain fatty acids into mitochondria and fatty acid beta-oxidation. Initially, triglycerides are hydrolyzed into fatty acids and glycerol. Long-chain fatty acid chains are first linked to coenzyme $A(C \circ A)$, and then fatty acids are transferred to carnitine by the catalytic activity of the enzyme carnitine palmitoyltransferase 1B (CPT1B), which is anchored in the outer mitochondrial membrane. The complex of a fatty acid and carnitine (Acyl-carnitine) is transported across the mitochondrial membranes by a translocase, and the enzyme carnitine palmitoyltransferase 2 (CPT2), associated with the inner surface of the inner mitochondrial membrane, catalyzes the conversion of Acylcarnitine complexes into carnitine and Acyl-CoA. Acyl-CoA molecules are beta-oxidized by mitochondrial enzymes, generating Acetyl-CoA, which enters the Krebs' cycle. 2-cell embryos can predict which embryos will develop to the blastocyst stage (27).

Pyruvate metabolism can also be predictive of embryo quality, as demonstrated by Guerif et al. (33). Of all substrates examined by the authors, pyruvate consumption in intermediary metabolism was consistently related to the morphological progression of embryos that develop in vitro compared to those that remained static (68 vs $13-25 \%$, respectively) (33).

As described above, a low metabolic rate is predictive of viability in in vitro embryos (the 'quiet metabolism' theory) and is related to blastocyst development, similar to that observed in in vivo embryos, which are naturally 'quiet' $(5,27)$. In other words, the fact that in vitro embryos do not show the well-known 'quiet' metabolism of in vivo embryos may compromise embryo viability $(5,5,34)$.

A large number, approximately $61 \%$ (1), of in vitro embryos do not develop after transfer to cows, and the calves that are born after transference may eventually develop anomalies related to the 'large offspring syndrome' $(4,6)$. This phenomenon suggests that phenotypic characteristics are affected by the culture system, leading to the conclusion that the in vitro culture conditions currently used for embryo development might trigger a stress response $(6,34)$.

In vitro oxidative rates are higher than those occurring in vivo and result in high levels of reactive oxygen species, thereby reducing embryonic development (35). The 'ideal' medium composition for in vitro embryo development should support adequate embryo morphologic development and cell replication rates at each embryo stage, allowing the birth of viable and healthy offspring after transfer (16) and minimizing the stress of cell culture in vitro (35).
In addition, the embryo culture medium currently utilized is not adequate to support embryonic requirements and not sufficiently similar to the conditions in the female reproductive tract. Uterine fluids have low levels of oxygen (3-5\%) and energetic substrates compared to embryo culture (21\%) (32), which can be considered a pro-oxidant environment. Also, while culture composition does not change in vitro (17), uterine fluid composition changes dynamically during embryonic development, probably due to changes in energetic requirements between embryo stages (14). Therefore, the ideal in vitro culture should mimic the in vivo medium, including nutrient composition and oxygen concentration.

\section{Concluding Remarks}

In the last few years, several publications $(9,11,14-$ $16,20,22,23,25,28-31)$ have addressed the energy demands of bovine embryos in vivo and in vitro, to establish ideal in vitro conditions for bovine embryonic development. Early development in vitro generates less viable embryos, and metabolic evidence suggests that this phenomenon is due to the induction of cellular stress by currently used embryo culture conditions.

The analysis of changes in culture medium composition during in vitro development can be used to evaluate embryo quality, and thus aid in the improvement of embryo culture systems, with the ultimate goal of producing high-quality bovine embryos $(35,36)$, providing increased rates of viable pregnancies, and increasing the production and quality of cattle.

Despite recent efforts to improve our understanding of the metabolism of early embryos, several important questions remain unanswered regarding the ideal concentration 
of energetic substrates, metabolic differences among preimplantation stages, the influence of substrate combinations, the importance of intracellular glycogen and triglyceride reservoirs, and how energetic supplements influence pregnancy rates. Addressing these questions is a necessary step towards the production of in vitro embryos of similar quality to those produced in vivo.

\section{References}

1. Seneda MM, Marinho LSR, Untura RM, Morotti F, Moino LL, Rigo AG, et al. Large-scale programs for recipients of in vitro-produced embryos. Anim Reprod 2012; 9: 323-328.

2. Santos JE, Cerri RL, Sartori R. Nutritional management of the donor cow. Theriogenology 2008; 69: 88-97, doi: 10.1016/j.theriogenology.2007.09.010.

3. Scientific Advisory Committee on Nutrition (SACN). The influence of maternal, fetal and child nutrition on the development of chronic disease in later life. 2011.

4. Farin CE, Farin PW, Piedrahita JA. Development of fetuses from in vitro-produced and cloned bovine embryos. J Anim Sci 2004; 82 (E-Suppl): E53-E62.

5. Leese HJ. Metabolism of the preimplantation embryo: 40 years on. Reproduction 2012; 143: 417-427, doi: 10.1530/ REP-11-0484.

6. Leese HJ. Quiet please, do not disturb: a hypothesis of embryo metabolism and viability. Bioessays 2002; 24: 845849, doi: 10.1002/bies.10137.

7. Gardner DK, Lane M, Stevens J, Schoolcraft WB. Noninvasive assessment of human embryo nutrient consumption as a measure of developmental potential. Fertil Steril 2001; 76: 1175-1180, doi: 10.1016/S0015-0282(01) 02888-6.

8. Brinster RL. Embryo development. J Anim Sci 1974; 38: 1003-1012.

9. Ferguson EM, Leese HJ. Triglyceride content of bovine oocytes and early embryos. J Reprod Fertil 1999; 116: 373378, doi: 10.1530/jrf.0.1160373.

10. Thompson JG, Bell ACS, Tervit HR. Partitioning of glucose carbon in post-compaction ovine embryos. Anim Reprod Sci 1995; 38: 119-126, doi: 10.1016/0378-4320(94)01350-U.

11. Sturmey RG, Reis A, Leese HJ, McEvoy TG. Role of fatty acids in energy provision during oocyte maturation and early embryo development. Reprod Domest Anim 2009; 44 (Suppl 3): 50-58, doi: 10.1111/j.1439-0531.2009.01402.x.

12. Wilding M, Coppola G, Dale B, Di Matteo L. Mitochondria and human preimplantation embryo development. Reproduction 2009; 137: 619-624, doi: 10.1530/REP-080444.

13. Sturmey RG, Leese HJ. Energy metabolism in pig oocytes and early embryos. Reproduction 2003; 126: 197-204, doi: 10.1530/rep.0.1260197.

14. Thompson JG, Partridge RJ, Houghton FD, Cox Cl, Leese $\mathrm{HJ}$. Oxygen uptake and carbohydrate metabolism by in vitro derived bovine embryos. J Reprod Fertil 1996; 106: 299306, doi: 10.1530/jrf.0.1060299.

15. Khurana NK, Niemann H. Energy metabolism in preimplantation bovine embryos derived in vitro or in vivo. Biol Reprod 2000; 62: 847-856, doi: 10.1095/biolreprod62.4.847.

\section{Acknowledgments}

The authors would like to thank Publicase Comunicações Científicas for English revision. We would also like to thank Dr. Henry J. Leese for suitable suggestions for the present manuscript.

16. Partridge RJ, Leese HJ. Consumption of amino acids by bovine preimplantation embryos. Reprod Fertil Dev 1996; 8: 945-950, doi: 10.1071/RD9960945.

17. Barnett DK, Bavister BD. What is the relationship between the metabolism of preimplantation embryos and their developmental competence? Mol Reprod Dev 1996; 43: 105-133, doi: 10.1002/(SICI)1098-2795(199601)43:1<1 05::AID-MRD13>3.0.CO;2-4.

18. Lane M, Gardner DK. Differential regulation of mouse embryo development and viability by amino acids. J Reprod Fertil 1997; 109: 153-164, doi: 10.1530/jrf.0.1090153.

19. Watson AJ, Barcroft LC. Regulation of blastocyst formation. Front Biosci 2001; 6: D708-D730, doi: 10.2741/Watson.

20. Donnay I, Leese HJ. Embryo metabolism during the expansion of the bovine blastocyst. Mol Reprod Dev 1999; 53: 171-178, doi: 10.1002/(SICl)1098-2795(199906)5 3:2<171::AID-MRD6>3.0.CO;2-F.

21. Bavister BD, Squirrell JM. Mitochondrial distribution and function in oocytes and early embryos. Hum Reprod 2000; 15 (Suppl 2): 189-198, doi: 10.1093/humrep/15.suppl_2. 189.

22. Houghton FD, Humpherson PG, Hawkhead JA, Hall CJ, Leese $\mathrm{HJ} . \mathrm{Na}^{+}, \mathrm{K}^{+}$, ATPase activity in the human and bovine preimplantation embryo. Dev Biol 2003; 263: 360366, doi: 10.1016/j.ydbio.2003.07.014.

23. Gopichandran N, Leese HJ. Metabolic characterization of the bovine blastocyst, inner cell mass, trophectoderm and blastocoel fluid. Reproduction 2003; 126: 299-308, doi: 10.1530/rep.0.1260299.

24. Houghton FD. Energy metabolism of the inner cell mass and trophectoderm of the mouse blastocyst. Differentiation 2006; 74: 11-18, doi: 10.1111/j.1432-0436.2006.00052.x.

25. Orsi NM, Leese HJ. Ammonium exposure and pyruvate affect the amino acid metabolism of bovine blastocysts in vitro. Reproduction 2004; 127: 131-140, doi: 10.1530/rep.1. 00031.

26. Thompson JG, Sherman AN, Allen NW, McGowan LT, Tervit HR. Total protein content and protein synthesis within pre-elongation stage bovine embryos. Mol Reprod Dev 1998; 50: 139-145, doi: 10.1002/(SICI)1098-2795(199806) 50:2<139::AID-MRD3>3.0.CO;2-L.

27. Sturmey RG, Bermejo-Alvarez $P$, Gutierrez-Adan A, Rizos $D$, Leese HJ, Lonergan P. Amino acid metabolism of bovine blastocysts: a biomarker of sex and viability. Mol Reprod Dev 2010; 77: 285-296, doi: 10.1002/mrd.21173.

28. Ferguson EM, Leese HJ. A potential role for triglyceride as an energy source during bovine oocyte maturation and early embryo development. Mol Reprod Dev 2006; 73: 11951201, doi: 10.1002/mrd.20494. 
29. Gomez E, Duque P, Diaz E, Diez C. Effects of acetoacetate on in vitro development of bovine embryos in medium containing citrate and myo-inositol. Reprod Domest Anim 2001; 36: 189-194, doi: 10.1046/j.1439-0531.2001.d01-35.x.

30. Gomez E, Duque P, Diaz E, Facal N, Antolin I, Hidalgo C, et al. Effects of acetoacetate and D-beta-hydroxybutyrate on bovine in vitro embryo development in serum-free medium. Theriogenology 2002; 57: 1551-1562, doi: 10.1016/S0093-691X(02)00660-X.

31. Sutton-McDowall ML, Feil D, Robker RL, Thompson JG, Dunning KR. Utilization of endogenous fatty acid stores for energy production in bovine preimplantation embryos. Theriogenology 2012; 77: 1632-1641, doi: 10.1016/j. theriogenology.2011.12.008.

32. Leese HJ, Baumann CG, Brison DR, McEvoy TG, Sturmey RG. Metabolism of the viable mammalian embryo: quietness revisited. Mol Hum Reprod 2008; 14: 667-672, doi: 10.1093/molehr/gan065.

33. Guerif $F$, McKeegan P, Leese HJ, Sturmey RG. A simple approach for COnsumption and RElease (CORE) analysis of metabolic activity in single mammalian embryos. PLoS One 2013; 8: e67834, doi: 10.1371/journal.pone.0067834.

34. Thompson JG. The impact of nutrition of the cumulus oocyte complex and embryo on subsequent development in ruminants. J Reprod Dev 2006; 52: 169-175, doi: 10.1262/ jrd.17089.

35. Leese HJ, Sturmey RG, Baumann CG, McEvoy TG. Embryo viability and metabolism: obeying the quiet rules. Hum Reprod 2007; 22: 3047-3050, doi: 10.1093/humrep/ dem253.

36. Urbanski JP, Johnson MT, Craig DD, Potter DL, Gardner DK, Thorsen T. Noninvasive metabolic profiling using microfluidics for analysis of single preimplantation embryos. Anal Chem 2008; 80: 6500-6507, doi: 10.1021/ac8010473. 